Cite this: Phys. Chem. Chem. Phys., 2013 15, 14248

Received 16th May 2013,

Accepted 2nd July 2013

DOI: $10.1039 / \mathrm{c3cp} 52061 \mathrm{~g}$

www.rsc.org/pccp

\title{
Manipulating spin hyper-polarization by means of adiabatic switching of a spin-locking RF-field
}

\author{
Alexey S. Kiryutin, ${ }^{\text {ab }}$ Konstantin L. Ivanov, ${ }^{\text {ab }}$ Alexandra V. Yurkovskaya, ${ }^{\text {ab }}$ \\ Hans-Martin Vieth ${ }^{c}$ and Nikita N. Lukzen ${ }^{\text {ab }}$
}

\begin{abstract}
We propose a technique for transferring the multiplet spin polarization (CIDNP or PHIP, or one created by any other method), which is the mutual entanglement of spins, into net hyper-polarization with respect to the direction of a high magnetic field by slowly (adiabatically) switching-off a strong external RF-field with a specially selected frequency. The net hyper-polarized molecules can then be used in NMR spectroscopy or imaging for strong signal enhancement.
\end{abstract}

\section{Introduction}

The level of development of Nuclear Magnetic Resonance (NMR) spectroscopy and imaging achieved during the last decades has made them particularly efficient and versatile tools for studying the structure and dynamics of molecules and living organisms. This results in the popularity of NMR spectroscopy and Magnetic Resonance Imaging (MRI) methods in physics, chemistry, biology and medicine. The limiting factor in NMR applications is frequently sensitivity, which is notoriously low because of the small interaction of nuclear spins with external magnetic fields. As a result the energy of nuclear spin transitions and therefore the detection sensitivity of the photons involved are low; for the same reason the population difference between nuclear spin states and thus the total magnetization under equilibrium conditions are tiny. A possible strategy for minimizing this inherent drawback of the NMR methods is utilizing spin systems, which are far off thermal equilibrium, i.e., hyper-polarized. Currently, several techniques are used to substantially increase the NMR sensitivity such as Dynamic Nuclear Polarization (DNP), ${ }^{1-3}$ Chemically Induced Nuclear Polarization (CIDNP), ${ }^{4}$ Para-Hydrogen Induced Polarization (PHIP) ${ }^{5-7}$ Optical Nuclear Polarization (ONP $)^{8,9}$ and Spin-Exchange Optical Pumping (SEOP). ${ }^{10}$

Particularly strong hyper-polarization is provided by the PHIP technique. PHIP is observed after catalytic addition of parahydrogen molecules (the $\mathrm{H}_{2}$ molecule in its singlet spin isomer) to molecules with double or triple $\mathrm{C}-\mathrm{C}$ bonds in such a way that the symmetry of the two protons is broken. The chemical

\footnotetext{
${ }^{a}$ International Tomography Center SB RAS, Institutskaya 3a, Novosibirsk 630090,

Russia. E-mail: ivanov@tomo.nsc.ru; Fax: +7 383 333-1399; Tel: +7 383 330-8868

${ }^{b}$ Novosibirsk State University, Pirogova 2, Novosibirsk 630090, Russia

${ }^{c}$ Freie Universität Berlin, Arnimallee 14, Berlin 14195, Germany
}

reaction thus serves to transfer the large spin alignment of the para- $\mathrm{H}_{2}$ molecule into an NMR observable. This creates unprecedented strong NMR signal enhancement because out of the 4 states of the $\mathrm{H}_{2}$ molecule (one singlet and three triplet), the singlet state is highly enriched at low temperatures. As a result, the method gives a gain in NMR signal amplitude by several orders of magnitude. For simplicity we assume in the following discussion $100 \%$ enrichment in para- $\mathrm{H}_{2}$. Up to now there are two conventional schemes for creation of PHIP-type hyper-polarization. The first scheme, the so-called PASADENA (Parahydrogen And Synthesis Allow Dramatically Enhanced Nuclear Alignment) experiment, ${ }^{11}$ corresponds to the situation where the hydrogenation reaction is carried out directly in the detection field of the NMR spectrometer, which yields multiplet polarization (mutual alignment of spins) of the two attached hydrogen atoms in the product of hydrogenation. In the second scheme, the ALTADENA (Adiabatic Longitudinal Transport After Dissociation Engenders Net Alignment) experiment, ${ }^{12}$ the hydrogenation reaction is run in the Earth's magnetic field with subsequent adiabatic transfer of the hyper-polarized product into the NMR spectrometer. As a consequence, net polarization of each of the two protons (though of opposite sign), which originated from the parahydrogen molecule, is formed. Despite the anomalous signal intensities of the individual NMR lines of about 10000 the net polarization of the product molecule as a whole is zero. Similar polarization patterns can also be obtained by means of the CIDNP technique when hyper-polarization is prepared at low fields. ${ }^{13}$ At the same time, in many CIDNP and PHIP applications it is highly desirable to have molecules with considerable net hyper-polarization, which can be difficult to achieve, for instance, by manipulating the ALTADENA or PASADENA pattern by NMR pulse sequences. Although there are successful examples of utilizing NMR pulses 
to control and transfer PHIP-derived spin order ${ }^{5,7,14-18}$ the need for precise synchronization of the pulses with the chemical reaction and control of coherences between the spin states can be a problem. To have at the output net hyper-polarization is especially crucial for MRI since at typical magnetic fields used in the MRI technique NMR multiplet structures as well as chemical shifts cannot be resolved with the consequence that only the integral net hyper-polarization is observable. A remedy ${ }^{19-22}$ is given by using spin echoes to obtain macroscopic magnetization even from multiplet-polarized spins; however, it is far easier to work with net-polarized spins.

In this work we propose a novel method of manipulating hyper-polarization that enables efficient transfer of multiplet spin order into net spin order. The method is relatively simple as it does not require complicated pulses sequences and allows one to transfer the initial multiplet spin order into almost any desired polarization pattern.

We propose that spin hyper-polarization is manipulated according to the experimental protocol depicted in Fig. 1, which comprises three consecutive stages. First, hyper-polarization is prepared in molecules experiencing the high external $B_{0}$ field of the NMR magnet $\left(B_{0}>1 \mathrm{~T}\right)$ and in addition a strong radiofrequency field $\left(\mathrm{RF}_{1}\right)$ providing spin locking conditions for the spin system (stage 1). After the polarization is prepared during the time period $\tau_{\mathrm{p}}$ the RF-field is switched-off during time $\tau_{\text {off }}$ in an adiabatic way (stage 2) and then the NMR spectrum is recorded (stage 3). Depending on the frequency
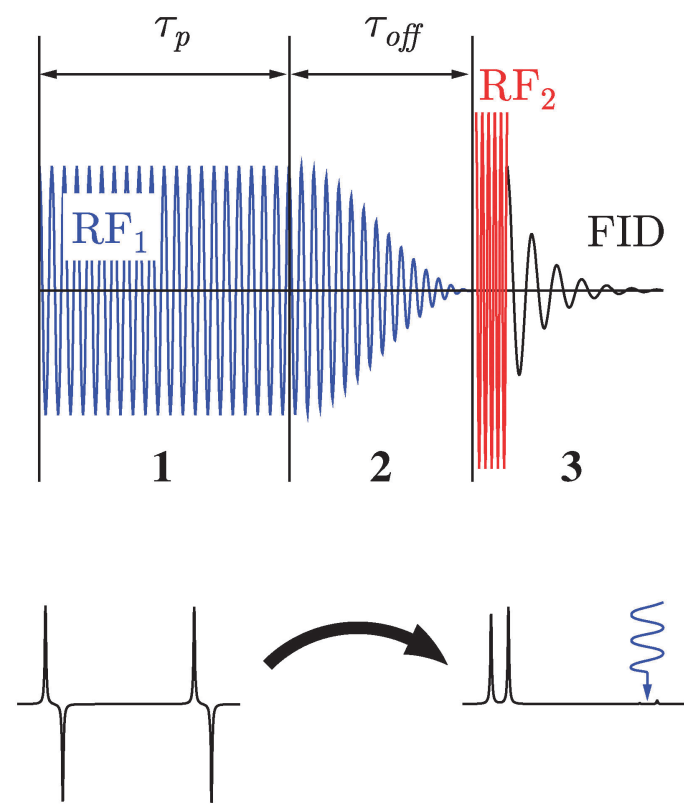

Fig. 1 Protocol of the hyper-polarization experiment in the presence of a strong RF-field, which is reduced to zero. Consecutive stages of the timing scheme: preparation of hyper-polarization in an external magnetic field $B_{0}$ and a strong RF-field, $\mathrm{RF}_{1}$ (stage 1); adiabatic fading of the $\mathrm{RF}_{1}$ field (stage 2); applying a nonselective RF-pulse, $\mathrm{RF}_{2}$, and detection of the Free Induction Decay (FID) (stage 3) to obtain the Fourier-Transform NMR spectrum. In the bottom part the transformation of the PASADENA spectral pattern (left) to the new pattern (right) is shown when the $\mathrm{RF}_{1}$ frequency coincides with the Larmor precession frequency of one of the spins. of the RF-field, $\nu_{\mathrm{rf}}$, one can get different spectral patterns of choice. In particular, initial multiplet polarization (which gives strongly enhanced individual lines but zero total intensity of the spectrum) can be converted into net polarization of the whole spin system. Whilst the use of multiplet hyper-polarization, although it is efficiently provided by CIDNP and PHIP techniques, is often not an option in NMR spectroscopy and imaging (despite high enhancement of individual NMR lines) net hyper-polarization can be used with standard pulse sequences thus straightforwardly giving signal enhancements of up to several orders of magnitude.

\section{Theory}

Here we will not discuss all theoretical details of the method but only give its main idea and show a few examples of how it works. The full theoretical treatment of the problem under consideration will be published elsewhere. Although the method is more general and works for multispin systems as well, for the sake of simplicity, we will discuss only a system of two scalar coupled spins. For the same reason we will assume that hyper-polarization is prepared by means of PHIP although the technique is also applicable to the CIDNP case.

We will prepare hyper-polarization in molecules experiencing the high external $B_{0}$ field of the NMR magnet and a strong RF-field providing spin locking conditions for the spin system. The spin system is described by the following Hamiltonian in the reference frame rotating with the frequency $\nu_{\mathrm{rf}}$ of the RF-field of amplitude $\nu_{1}^{0}$ :

$$
\hat{H}_{0}=-\left(\nu_{\mathrm{a}}-\nu_{\mathrm{rf}}\right) \hat{I}_{\mathrm{az}}-\left(\nu_{\mathrm{b}}-\nu_{\mathrm{rf}}\right) \hat{I}_{\mathrm{bz}}+J\left(\hat{\mathbf{I}}_{\mathrm{a}} \cdot \hat{\mathbf{I}}_{\mathrm{b}}\right)+\nu_{1}^{0}\left(\hat{I}_{\mathrm{ax}}+\hat{I}_{\mathrm{bx}}\right) .
$$

Here $\nu_{\mathrm{a}}$ and $\nu_{\mathrm{b}}$ are the precession frequencies of the spins given in the $\delta$-scale of chemical shift; $J$ is the spin-spin interaction of the spins. During a sufficiently long preparation period the eigen-states of the Hamiltonian $\hat{H}_{0}$ acquire non-thermal population in accordance with the character of polarization, while the coherences between them are washed out. ${ }^{23}$ We also assume that the RF-field is sufficiently strong, i.e.,

$$
\nu_{1}^{0} \gg\left(\nu_{\mathrm{a}}-\nu_{\mathrm{rf}}\right),\left(\nu_{\mathrm{b}}-\nu_{\mathrm{rf}}\right)
$$

and moreover

$$
\frac{\left(\nu_{\mathrm{a}}-\nu_{\mathrm{rf}}\right)^{2}-\left(\nu_{\mathrm{b}}-\nu_{\mathrm{rf}}\right)^{2}}{2 \nu_{1}^{0}} \ll J .
$$

These conditions guarantee that in the presence of the RF-field the eigen-states of the Hamiltonian are characterized by a particular total spin (singlet and triplet states in our case). After the polarization is prepared the RF-field is switched-off during time $\tau_{\text {off }}$ and subsequently the NMR spectrum is recorded. Thus, the evolution of the spin system proceeds according to the following term in the Hamiltonian:

$$
\nu_{1}(t)\left(\hat{I}_{\mathrm{ax}}+\hat{I}_{\mathrm{bx}}\right)
$$


where $\nu_{1}(t=0)=\nu_{1}^{0}$ and $\nu_{1}\left(t=\tau_{\text {off }}\right)=0$. We will also assume that in the absence of the RF-field the spins are weakly coupled: $|J| \ll\left|\nu_{\mathrm{a}}-\nu_{\mathrm{b}}\right|$ in order to make the analysis simpler.

The main assumption for the theoretical analysis is that the switching is considered slow (adiabatic) so that the populations of the coupled levels have enough time to adjust themselves to the changes of the Hamiltonian (1) (populations follow the energy levels); hence populations can follow the spin states during the switching. The case of sudden field change is of no interest because it leads to the usual PASADENA pattern (although due to the fast switching zero-quantum coherences can be formed, which give unusual NMR lines ${ }^{24}$ ). In the general case of RF-field switching only a numerical solution of the problem under study is possible.

At sufficiently high $\nu_{1}^{0}$ RF-field before it is turned off only one spin eigen-state (singlet state) out of four gets populated. ${ }^{24}$ When $J>0$ this state is always the second lowest state in energy. As a result of the subsequent switching its population equal to $P_{\mathrm{S}}$ selectively goes to only one state (in the case of degeneracy to only two states) of the Hamiltonian that is valid in the absence of the RF-field. Let us now discuss what spin states can acquire population under different conditions. Before performing the correlation of spin states at high $\nu_{1}$ and at $\nu_{1}\left(t=\tau_{\text {off }}\right)=0$, i.e., keeping the order in accordance with their energy, it is important to note that in the rotating frame the spin states can be ordered differently as compared to the laboratory frame. Although at $\nu_{1}\left(t=\tau_{\text {off }}\right)=0$ the eigen-states of the system are still the product states of the individual spins, their order can change depending on $\nu_{\mathrm{rf}}$. When $\nu_{1}=0$ the energies of the eigen-states of $\hat{H}_{0}$ are:

$$
\begin{gathered}
E_{\alpha \alpha}=-\frac{\delta \nu_{\mathrm{a}}}{2}-\frac{\delta \nu_{\mathrm{b}}}{2}+\frac{J}{4}, E_{\alpha \beta}=-\frac{\delta \nu_{\mathrm{a}}}{2}+\frac{\delta \nu_{\mathrm{b}}}{2}-\frac{J}{4}, \\
E_{\beta \alpha}=\frac{\delta \nu_{\mathrm{a}}}{2}-\frac{\delta \nu_{\mathrm{b}}}{2}-\frac{J}{4}, E_{\beta \beta}=\frac{\delta \nu_{\mathrm{a}}}{2}+\frac{\delta \nu_{\mathrm{b}}}{2}+\frac{J}{4}
\end{gathered}
$$

Only when $\delta \nu_{\mathrm{a}}=\left(\nu_{\mathrm{a}}-\nu_{\mathrm{rf}}\right)$ and $\delta \nu_{\mathrm{b}}=\left(\nu_{\mathrm{b}}-\nu_{\mathrm{rf}}\right)$ are of the same sign ( $\nu_{\mathrm{rf}}$ is located "outside" the NMR spectrum of the spin system) will the $\alpha \alpha$ and $\beta \beta$ states have the highest and lowest energies. (As usual, $\alpha$ and $\beta$ stand for the spin-up and spindown states of spin 1/2.) In all other cases the spin energy levels are grouped in different order. Different cases are shown in Fig. 2 with the corresponding correlation diagram of the energy levels and the NMR spectra after adiabatic switching of the RF-field. The spectral pattern can be conveniently interpreted in terms of the net polarization of individual spins, $\left\langle I_{\mathrm{az}}\right\rangle$ and $\left\langle I_{\mathrm{bz}}\right\rangle$ and the multiplet polarization, $\left\langle I_{\mathrm{az}} I_{\mathrm{bz}}\right\rangle$. They are the expectation values of the corresponding spin operators:

$$
\left\langle I_{\mathrm{az}}\right\rangle=\operatorname{Tr}\left\{\hat{I}_{\mathrm{az}} \hat{\rho}\right\},\left\langle I_{\mathrm{bz}}\right\rangle=\operatorname{Tr}\left\{\hat{I}_{\mathrm{bz}} \hat{\rho}\right\},\left\langle I_{\mathrm{az}} I_{\mathrm{bz}}\right\rangle=\operatorname{Tr}\left\{\hat{I}_{\mathrm{az}} \hat{I}_{\mathrm{bz}} \hat{\rho}\right\}
$$

Here $\hat{\rho}$ is the density matrix of the spin system after the RF-field is turned off. Contributions of different spin orders can be easily obtained from the spectrum: net polarization of each spin is the integral over the corresponding NMR multiplet, i.e., over all NMR lines, which correspond to the transitions of this spin; multiplet polarization gives the difference in intensity of the NMR lines within the multiplet. An interesting feature of
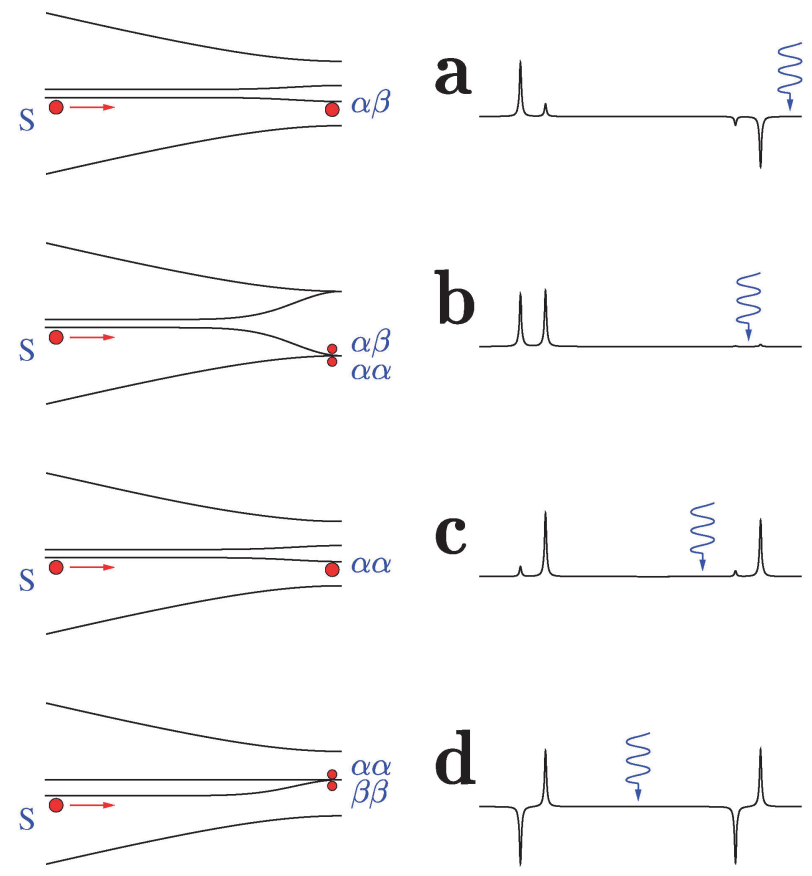

strong $\mathrm{B}_{1}$

$\mathrm{B}_{1}=0$

Chemical shift

Fig. 2 Correlation diagrams of the energy levels of the Hamiltonian (1) of the two-spin system during fading of the strong RF-field at different $\nu_{\text {rf }}$ (left) and the corresponding PHIP spectra (right). Positions of $\nu_{\mathrm{rf}}$ with respect to the Larmor precession frequencies of spins $\nu_{\mathrm{a}}$ and $\nu_{\mathrm{b}}$ are shown on the spectra by blue arrows. Populated energy levels in the presence of strong RF-field (the singlet state is the second lowest in energy) and at $\nu_{1}=0$ (order of levels is in accordance with eqn (5)) are indicated; size of the balls corresponds to the amount of population of the levels. Cases $(a-d)$ correspond to different $\nu_{\mathrm{rf}}$ positions.

the hyper-polarized spin systems is the non-trivial dependence 25,26 of the line intensity on the rotation angle, $\varphi$, of the RF-pulse, which is used to detect the Fourier-Transform NMR spectrum. While the contributions of the net polarization are proportional to $\sin \varphi$, those coming from the multiplet polarization are proportional to $\sin 2 \varphi$. Therefore, using $\pi / 2$-pulses for detection is not always optimal as contributions from multiplet polarization are suppressed. Flip angle dependent NMR spectra can be calculated in the same way as before. ${ }^{26,27}$

In particular, the spectral pattern is conditioned by the quantities $\delta \nu_{\mathrm{a}}$ and $\delta \nu_{\mathrm{b}}$. For clarity, let us assume that spin "a" has a larger chemical shift: $\left(\nu_{\mathrm{a}}-\nu_{\mathrm{b}}\right)=\left(\delta \nu_{\mathrm{a}}-\delta \nu_{\mathrm{b}}\right)>0$. For the two-spin system there are the following cases, in which the spectral shape after turning off the RF-field is remarkably different:

(1) Both quantities $\nu_{\mathrm{a}}$ and $\nu_{\mathrm{b}}$ have the same sign, i.e., the frequency $\nu_{\mathrm{rf}}$ is located "outside" the spectrum of the coupled spin system. In this case all the spin states are non-degenerate at any $\nu_{1}$ value and therefore one has to correlate the singlet state (second lowest in energy) at $\nu_{1}(t=0)=\nu_{1}^{0}$ with the second lowest state at $\nu_{1}=0$. The latter state is the $\alpha \beta$ state, which acquires a population equal to $P_{\mathrm{S}}$, whereas the other three states stay empty. In such a situation the spectrum has exactly the same appearance as the ALTADENA spectrum: both spins acquire net polarization of the same amplitude but of opposite 
sign; multiplet polarization is also present. The amplitude of the net polarization, $\left\langle I_{\mathrm{az}}\right\rangle$ and $\left\langle I_{\mathrm{bz}}\right\rangle$, is equal to $P_{\mathrm{S}} / 2$; multiplet polarization of the spins, $\left\langle I_{\mathrm{az}} I_{\mathrm{bz}}\right\rangle$, is equal to $-P_{\mathrm{S}} / 4$. The corresponding level diagram and NMR spectra are shown in Fig. 2a.

(2) Frequency $\nu_{\mathrm{rf}}$ coincides with the Larmor frequency of one of the spins. In this situation there is a degeneracy of states because in the rotating frame the precession frequency of that spin is equal to zero. As a consequence, the initial singlet state population is shared evenly between a pair of states, which have lowest energy (Fig. 2b). In this case, the spin with Larmor frequency equal to $\nu_{\mathrm{rf}}$ has zero net polarization, while the other one is net polarized with the magnitude of the net polarization being equal to $P_{\mathrm{S}} / 2$ (see Fig. 2b). Multiplet polarization is zero in this case. Thus, the system acquires net polarization, which can be negative or positive depending on the frequency position: when $\nu_{\mathrm{a}}=\nu_{\mathrm{rf}}$ we obtain $\left\langle I_{\mathrm{az}}\right\rangle=0$ and $\left\langle I_{\mathrm{bz}}\right\rangle=-P_{\mathrm{S}} / 2$; when $\nu_{\mathrm{b}}=\nu_{\mathrm{rf}}$ then $\left\langle I_{\mathrm{az}}\right\rangle=P_{\mathrm{S}} / 2$ and $\left\langle I_{\mathrm{bz}}\right\rangle=0$.

(3) The quantities $\nu_{\mathrm{a}}$ and $\nu_{\mathrm{b}}$ are non-zero and have opposite signs, i.e., the frequency $\nu_{\mathrm{rf}}$ is located "inside" the NMR spectrum of the system. Different signs of $\delta \nu_{\mathrm{a}}$ and $\delta \nu_{\mathrm{b}}$ lead to a situation where the singlet state is correlated with the $\alpha \alpha$ and $\beta \beta$ states or with their combination. As a consequence, it becomes possible to populate these states in a selective way, which results in net polarization of the spin system. In this situation the spectral pattern changes drastically and there are three different situations:

(a) The frequency of the RF-field is closer to that of the spin with smaller chemical shift, i.e., $\left|\delta \nu_{\mathrm{a}}\right|>\left|\delta \nu_{\mathrm{b}}\right|$. Such a case is shown in Fig. 2c; the chosen set of parameters makes the $\alpha \alpha$ state the second lowest in energy and it selectively acquires the population of $P_{\mathrm{S}}$ after turning off the RF-field. As a consequence, both spins acquire positive net polarization: $\left\langle I_{\mathrm{az}}\right\rangle=\left\langle I_{\mathrm{bz}}\right\rangle=P_{\mathrm{S}} / 2$; in addition positive multiplet polarization is present, which is equal to $\left\langle I_{\mathrm{az}} I_{\mathrm{bz}}\right\rangle=P_{\mathrm{S}} / 4$.

(b) The RF-frequency is located exactly at the center of the NMR spectrum: $\nu_{\mathrm{rf}}=\left(\nu_{\mathrm{a}}+\nu_{\mathrm{b}}\right) / 2$. This choice of parameters results in degeneracy of the $\alpha \alpha$ and $\beta \beta$ states, both of which acquire populations of $P_{\mathrm{S}} / 2$ from the initially populated spin state (Fig. 2d). In this situation the spectrum, at a first glance, reminds of the PASADENA spectrum. Indeed, the net polarization of both spins is equal to zero and only multiplet polarization is present. However, the multiplet polarization is positive: $\left\langle I_{\mathrm{az}} I_{\mathrm{bz}}\right\rangle=P_{\mathrm{S}} / 4$, while in the PASADENA case it is always negative.

(c) The RF-frequency is closer to the spin with the larger chemical shift, i.e., $\left|\delta \nu_{\mathrm{a}}\right|<\left|\delta \nu_{\mathrm{b}}\right|$. In this case only the $\beta \beta$ state is populated after turning off the RF-field and both spins acquire negative net polarization: $\left\langle I_{\mathrm{az}}\right\rangle=\left\langle I_{\mathrm{bz}}\right\rangle=-P_{\mathrm{S}} / 2$; positive multiplet polarization is also present, $\left\langle I_{\mathrm{az}} I_{\mathrm{bz}}\right\rangle=P_{\mathrm{S}} / 4$.

Thus, by properly setting the RF-frequency one can obtain different spin order from the initially populated singlet state. It is the $\hat{I}_{\mathrm{x}}$ operator interacting with the time-dependent RF-field, which causes a change of the spin order, see eqn (4). However, correlating spin energy levels of the Hamiltonian (1) at strong RF-field and in the absence of the RF-field provides a more vivid picture of how the spin order is changed during the fading of the RF-field. The spectra shown in Fig. 2 are in perfect agreement with the numerically calculated spectra (not shown here) once a sufficiently long time $\tau_{\text {off }}$ is taken.

While Fig. 2 illustrates the concept of how the correlation of adiabatic states has to be performed and gives a few examples, all different cases, which are relevant for the two-spin system, are summarized in Fig. 3. When the frequency of the RF-field is placed "outside" the NMR spectrum of the spins an ALTADENA-like pattern is always observed independent of whether $\delta \nu_{\mathrm{a}}, \delta \nu_{\mathrm{b}}>0$ or $\delta \nu_{\mathrm{a}}, \delta \nu_{\mathrm{b}}<0$. When $\nu_{\mathrm{rf}}$ coincides with $\nu_{\mathrm{a}}$ or $\nu_{\mathrm{b}}$ the corresponding spin always has zero polarization and the multiplet polarization is also zero. The other spin then has either absorptive or emissive net polarization: for $\nu_{\mathrm{a}}>\nu_{\mathrm{b}}=\nu_{\mathrm{rf}}$ the "a" spin has positive net polarization, while for $\nu_{\mathrm{rf}}=\nu_{\mathrm{a}}>\nu_{\mathrm{b}}$ the "b" spin has negative net polarization. For $\nu_{\mathrm{rf}}$ placed "inside" the NMR spectrum the spins always get net polarized with only one exception: when $\nu_{\mathrm{rf}}=\left(\nu_{\mathrm{a}}+\nu_{\mathrm{b}}\right) / 2$ a PASADENA-type spectrum is observed, but with the opposite (positive) sign of the multiplet polarization as compared to the classical PASADENA case.

Thus, these qualitative results demonstrate that one can manipulate the initial polarization, which originally has the character of zero total net polarization. The method proposed allows one to convert this polarization into almost any kind of spin order in a well-defined way. The method does not require applying a sophisticated pulse sequence to manipulate the polarization. After the switching there is conversion of the spin order but not a reduction of spin order, i.e., there is no loss of hyper-polarization in our method. As a consequence, the intensities of the individual lines are the same as in the PASADENA

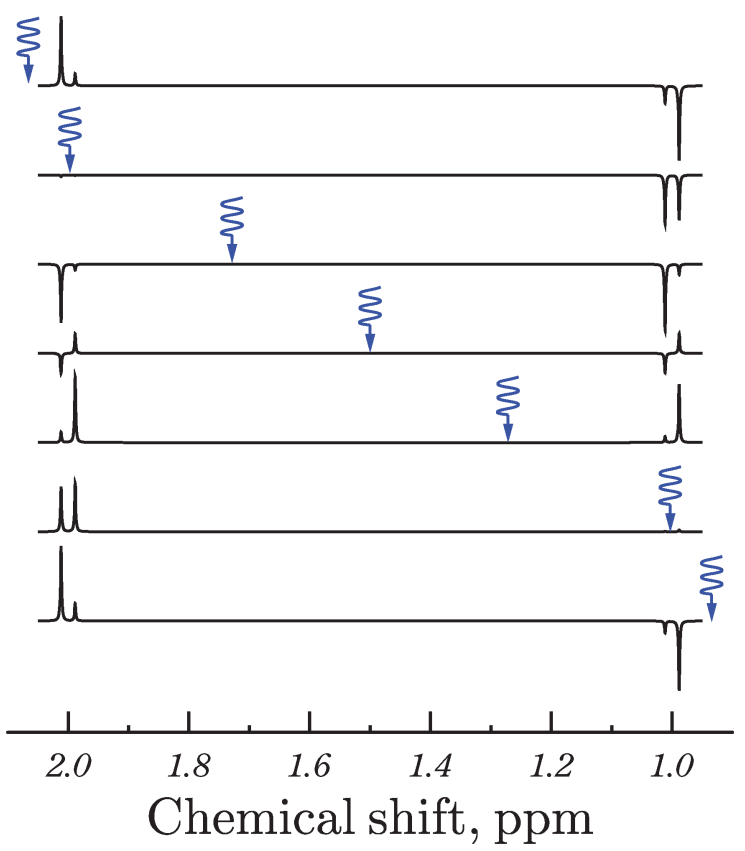

Fig. 3 Calculated PHIP spectra of the two-spin system after adiabatic fading of the strong RF-field at different $\nu_{\mathrm{rf}}$. Positions of $\nu_{\mathrm{rf}}$ with respect to the Larmor precession frequencies of spins $\nu_{\mathrm{a}}$ and $\nu_{\mathrm{b}}$ are shown on the spectra by blue arrows. Parameters of calculation: NMR frequency is $300 \mathrm{MHz}$, chemical shifts are $\delta_{\mathrm{a}}=2 \mathrm{ppm}$ and $\delta_{\mathrm{b}}=1 \mathrm{ppm}$; J-coupling is $4 \mathrm{~Hz}$; the locking field is switched off from $\nu_{1}^{0}=100 \mathrm{kHz}$ to zero in an adiabatic way; spin relaxation is neglected. 
case, but the lines do not cancel each other anymore. In general, our technique can always convert multiplet spin order into net spin order. The necessary conditions for applying the technique are as follows: (i) a sufficiently strong RF-field in accordance with eqn (2) and (3); (ii) turning off the RF-field is done adiabatically, however, faster than the system relaxes to thermal equilibrium. At the same time, the preparation times, at which the hyper-polarization process saturates, can be much longer than the $T_{1}$ and $T_{2}$ relaxation times because the spinlocking field isolates the singlet state, which can be long-lived (vide infra). This potentially allows one to accumulate a higher level of hyper-polarization as compared to the standard PASADENA experiment.

We performed numerical calculations to investigate the dependence of the spectra on the RF-field amplitude, frequency position and the time profile of turning it off. We analyzed two profiles of fading taking the same parameters of the spin system as those used in Fig. 3. Profile1 is the linear decrease of $\nu_{1}$ to zero, thus, the rate, $\mathrm{d} \nu_{1} / \mathrm{d} t$, of fading is constant. Profile 2 is the function $\nu_{1}(t)=\nu_{1}^{0} \cos ^{2}\left(\pi t / 2 \tau_{\text {off }}\right)$ with $\mathrm{d} \nu_{1} / \mathrm{d} t$ being equal to zero at $t=0$ and $t=\tau_{\text {off }}$. Our analysis shows for typical NMR parameters that even a relatively short time of RF switching provides nearly the same spectral pattern as for the adiabatic switching (the case of the longest switching time of $10 \mathrm{~s}$ ). For profile 1 even at $\tau_{\text {off }}=0.3 \mathrm{~s}$ the spectrum is nearly the same as in the adiabatic case, whereas for the smoother profile2 switching is very close to the adiabatic case even at $\tau_{\text {off }}=0.1 \mathrm{~s}$ and is purely adiabatic at $0.3 \mathrm{~s}$. As far as the amplitude of the RF-field is concerned, our numerical results show that $\nu_{1}$ of several $\mathrm{kHz}$ is usually sufficient for our purpose; $\nu_{1}=10 \mathrm{kHz}$ will be sufficient in all cases. Such amplitudes of the RF-fields, which can be kept for several seconds, are available in modern NMR spectrometers; heating of the sample is not a limiting factor either for the proposed technique at such a level of RF power.

Our results also show that our method can also be applied for manipulating hyper-polarization formed by other techniques, which produce a different kind of spin order. For example, one can polarize the spins by the CIDNP technique in such a way that they have multiplet polarization. The technique also works for a higher number of spins as was checked by numerical calculations for three-spin systems polarized by means of PHIP. In this situation one can obtain efficient transfer of PHIP to other protons in the molecules, because all protons are strongly coupled in the spin-locking field. By varying the frequency $\nu_{\mathrm{rf}}$ one can obtain almost any desired spectral pattern; in particular, one can form non-zero total net polarization, which is the variant most useful for applying hyper-polarization.

Another advantage of the technique is that in presence of a strong RF-field one can selectively populate so-called long-lived spin states (LLSs) ${ }^{28-30}$ by means of PHIP. The polarization "sustainability interval", ${ }^{31} T_{\text {LLS }}$, is much wider, when such a state is selectively populated, as compared to the longitudinal $T_{1}$-relaxation time. Since the ratio of maximal achievable polarization accumulated in LLSs and in longitudinal order is related to $T_{\text {LLS }}$ and $T_{1}$, one can accumulate a much higher stationary polarization. Polarization accumulated in LLSs always has a multiplet contribution; however, it can be converted into net polarization by subsequent adiabatic field switching. Accumulating polarization in presence of a strong RF-field has the additional advantage that all NMR signals from thermally polarized molecules are considerably reduced because at $\tau_{\mathrm{p}}>$ $T_{1}, T_{2}$ the corresponding NMR transitions are saturated by the RF-field.

\section{Experimental results}

\section{A. Sample preparation}

Catalyst [1,4-bis(diphenylphosphino)butane] (1,5-cyclooctadiene)rhodium(I) tetrafluoroborate $(98 \%)$ and deuterated styrene- $\mathrm{d}_{8}$ $(98 \%$ atom D) were received from Sigma-Aldrich. The $0.7 \mathrm{ml}$ solution containing $0.5 \mathrm{M}$ of styrene- $\mathrm{d}_{8}$ and $2.5 \mathrm{mM}$ of the catalyst was prepared by dissolution of the compounds in methanol- $\mathrm{d}_{4}$ (Deutero $\mathrm{GmbH}$ ) in the NMR-tube without any additional purification. PHIP was formed in the catalytic hydrogenation reaction of styrene to produce ethylbenzene. ${ }^{32}$ To create PHIP samples were purged with di-hydrogen gas enriched in its para-component (50\% enrichment) in a special $5 \mathrm{~mm}$ glass NMR tube in the NMR probe. ${ }^{33}$ At the bottom of the NMR sample tube a piece of sintered glass was inserted. This construction allows one to bubble gas from the bottom. After 15 seconds of bubbling the catalytic hydrogenation reaction (see Chart 1) proceeded for 16 seconds before the detection pulse. Since the styrene- $\mathrm{d}_{8}$ molecule was fully deuterated there are only two coupled protons in the product ethylbenzene. Thus, the spin system under investigation exactly corresponds to the situation described in the theoretical session. In the spectra we observed two polarized products: free ethylbenzene molecules (product A) and ethylbenzene forming a complex with the catalyst (product B). Spectra of both products look very similar to each other and are only shifted with respect to each other.

\section{B. PHIP in the presence of an RF-field of variable amplitude}

Our experiments revealed that the rate of producing hyperpolarized ethylbenzene was constant in the time interval of 15-30 seconds. Therefore, the preparation of PHIP under spin locking was performed in the following way: the cycle of one experiment started with a delay of 20 seconds (to destroy spin polarization formed during the previous cycle), after this delay the strong $\mathrm{RF}_{1}$ field $(10 \mathrm{kHz})$ was applied simultaneously with bubbling. After 15 seconds of bubbling the irradiation of $\mathrm{RF}_{1}$ continued for another 15 seconds; subsequently it was switched

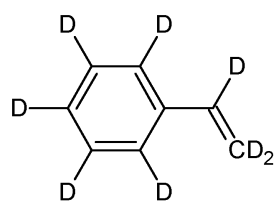

styrene- $\mathrm{d}_{8}$

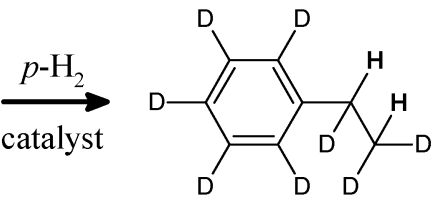

ethylbenzene- $\mathrm{d}_{8}$
Chart 1 Scheme of hydrogenation of styrene- $\mathrm{d}_{8}$ by para- $\mathrm{H}_{2}$ producing ${ }^{32}$ hyperpolarized ethylbenzene. 
off slowly during 1 second ( $\left.\tau_{\text {off }}\right)$. The RF detection pulse was applied in the next step with a short delay of about 50 milliseconds due to technical reasons (mechanical switching of a relay was used to switch between two RF power amplifiers). Acquisition of the FID during about 4 seconds finished one cycle of the experiment. The experiment was then repeated with a different RF-frequency, $\nu_{\mathrm{rf}}$. We used profile 2 for $\mathrm{RF}_{1}$ fading.

The following hardware was used to apply the strong RF-field and combine it with high-resolution NMR detection. From the NMR console TTL pulses were sent to the frequency generator PTS1, which created the input pulses with a carrier frequency of about $300 \mathrm{MHz}$ for the high-power amplifier HP1 (Class AB amplifier, KALMUS, $500 \mathrm{~W}$ Pulse, $150 \mathrm{~W}$ CW). The output signal of HP1 was used for the RF-pulse in NMR detection. In addition, from the NMR console gating TTL pulses were sent to the Pulse Delay Generator (PDG, Stanford Model DG535). Two channels of the PDG were used. From the first channel a long TTL pulse was obtained to operate a relay. It allowed us to switch between the two power sources used for $300 \mathrm{MHz}$ NMR detection (signal created by HP1) and for applying the strong RF-field (signal created by HP2, see text below). From the second channel of the PDG a trigger pulse was sent to the input of an arbitrary waveform generator (AWG) (AWG2021, Sony Tektronix). At the output of the AWG we obtained the envelope of the RF-field, i.e., the time profile of this field: a constant field for the preparation step and a decreasing field for the switching step performed by means of two voltage variable attenuators (Mini-Circuits model ZX732500-S+) in series. They shape the output amplitude of the frequency generator PTS2 operating at a carrier frequency of $\nu_{\mathrm{rf}}$ and driving the high-power amplifier HP2 (Class A broadband linear amplifier, Electronic Navigation Industries Inc. (ENI) Model 5100L), whose output signal was used to perform the spinlocking. After the RF-field is turned off (at the end of stage 2) the relay was switched from power amplifier HP2 to HP1. Finally, the RF-pulse created by HP1 was applied (stage 3) and the highresolution NMR spectrum was recorded.

\section{Results and discussion}

In the absence of the RF-field the polarization patterns were typical PASADENA patterns (the first spectrum in Fig. 4) for both products, $\mathrm{A}$ and $\mathrm{B}$. The presence of the RF-field changes the spectral patterns drastically when $\nu_{1}^{0}$ is sufficiently large (10 kHz in our case) to guarantee conditions (2) and (3) and $\tau_{\text {off }}$ is long enough ( $\tau_{\text {off }}=1 \mathrm{~s}$ ) to make the changes of Hamiltonian (1) adiabatic. These values are in accordance with our simulations.

By applying the strong RF-field and systematically varying its frequency we were able to obtain all the spectral patterns expected from the theoretical considerations (Fig. 2 and 3). We have performed a large number of experiments by systematically varying the RF-field frequency. The most representative PHIP spectra are shown in Fig. 4 presenting all the cases predicted by the theoretical treatment of the problem. When $\nu_{\mathrm{rf}}$ was outside the spectrum of the spin system the resulting PHIP spectrum exactly coincided with the ALTADENA spectral pattern (see the spectrum obtained, e.g., for $\nu_{\mathrm{rf}}=2.64 \mathrm{ppm}$ ). It is

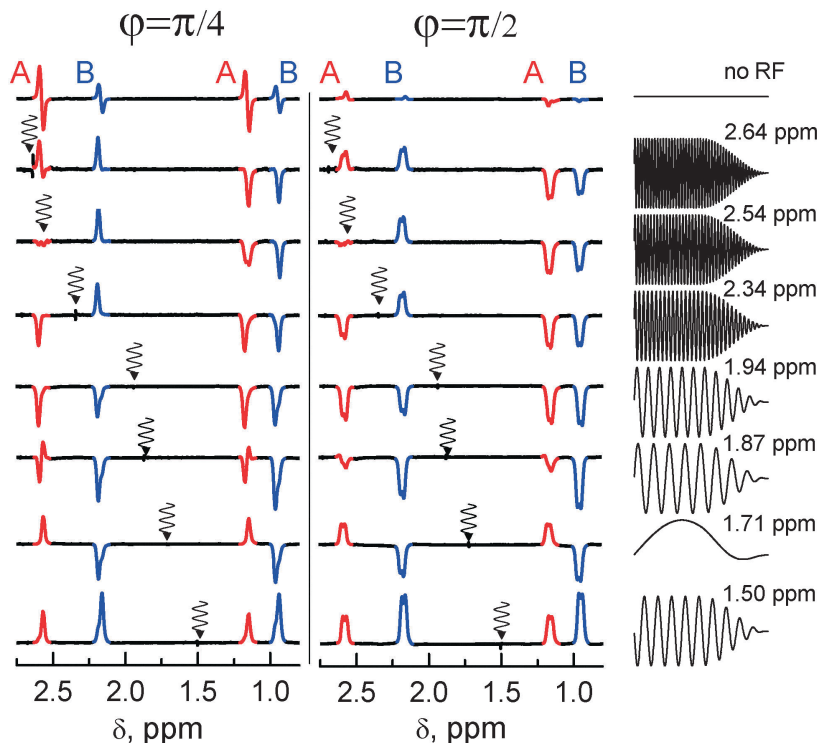

Fig. 4 Experimentally observed PHIP spectra of the two-spin system after adiabatic turning off the strong RF-field at different $\nu_{\mathrm{rf}}$. The position of $\nu_{\mathrm{rf}}$ with respect to the Larmor precession frequencies of spins $\nu_{\mathrm{a}}$ and $\nu_{\mathrm{b}}$ is shown on the spectra by black arrows; spectral lines shown in red and blue correspond to product $A$ and product $B$, respectively. The NMR frequency is $300 \mathrm{MHz}$. Spectra were detected using $\pi / 4$-pulses (left) and $\pi / 2$-pulses (right). In the very right the $\nu_{1}(t)$ time profiles are shown; $\nu_{\mathrm{rf}}$ is referenced to the RF-frequency corresponding to $1.7 \mathrm{ppm}$ in the $300 \mathrm{MHz}$ proton NMR spectrum.

also possible to net-polarize both spins (the polarization sign is reliably controlled by setting the RF-frequency: for instance, NMR lines are all positive at $\nu_{\mathrm{rf}}=1.50 \mathrm{ppm}$ and are all negative at $\nu_{\mathrm{rf}}=1.94 \mathrm{ppm}$ ), to net-polarize only one spin (irradiating on the frequency of the other one, see the spectrum of product A obtained for $\nu_{\mathrm{rf}}=2.54 \mathrm{ppm}$ ), and to obtain spectra, which look like the inverted PASADENA-spectra (see the spectrum of product A obtained for $\nu_{\mathrm{rf}}=1.87 \mathrm{ppm}$ ).

Thus, we were able to reproduce experimentally all the cases predicted by theory. This demonstrates that the method is feasible and can be realized with standard NMR equipment.

\section{Conclusions}

A new method has been proposed allowing one to convert initial multiplet spin order into polarization of almost any desired kind. It is based on hyper-polarizing a system of scalar coupled spins in the presence of a strong RF-field, which is subsequently slowly (adiabatically) reduced to zero. The technique can be useful for manipulating hyper-polarization, in particular, in cases where multiplet spin order gives the main contribution to hyper-polarization. The method allows one to not only reverse the sign of the multiplet polarization but also convert it into net hyper-polarization without any loss of the spin order. In contrast to multiplet spin order the contributions coming from net polarization do not disappear in the NMR spectrum in the presence of line broadening. Therefore net hyper-polarized signals can be used in NMR spectroscopy and imaging in combination with standard pulse sequences. 
For instance, our technique can be applied to the case of PHIP, which gives very strong hyper-polarization resulting in NMR enhancements of up to 10000 ; in contrast, the original polarization is purely of the multiplet kind. For the two-spin system we were able to show theoretically and experimentally that PHIP can be fully converted into net spin order: for instance, by properly setting the RF-field frequency one can convert the initial state population pattern into one where only the $\alpha \alpha$ and $\beta \beta$ spin states are populated; therefore both spins will get net polarized. In principle, our technique can also be realized by letting the liquid containing polarized molecules flow from a zone with strong RF-field with properly chosen frequency to a zone without RF-field at a speed, which provides adiabatic fading of the RF-field experienced by the spins. This can be advantageous for some applications, which use flow systems to deliver hyperpolarized molecules to the NMR detection position.

Another advantage of the proposed method is that using strong RF-fields potentially allows one to populate selectively the so-called long-lived spin states. The advantage of using LLSs is twofold: (i) hyper-polarization can be stored in such states for much longer times and (ii) by accumulating polarization over wider time intervals one can produce much higher levels before saturation is reached. Using strong RF-fields also enables considerable suppression of NMR signals of all thermally polarized molecules, which is advantageous in many applications. Our method can also be applied in the opposite way: initial net polarization can be converted into long-lived polarization by adiabatically switching on a strong RF-field with a properly set frequency thus populating the LLS. It is important to emphasize that spin-locking is often used in magnetic resonance spectroscopy; the speciality of our work is introducing adiabatic variation of the spin-lock field for selective manipulation of spin hyper-polarization.

Finally, it is worth noting that the method is not limited to manipulating PHIP only, but is applicable to other hyperpolarization techniques, for instance, to CIDNP as follows from our model calculations (not shown here). The method can also be extended to higher-spin systems, which initially carry only multiplet polarization, thus allowing one to create net hyperpolarization. Both statements have been confirmed by calculations and experiments; however, a detailed discussion of these aspects is the subject of an extended publication.

\section{Acknowledgements}

Financial support by the Russian Foundation for Basic Research (projects no. 12-03-31042, 12-03-33082, 12-03-31775, 13-03-00437), the Alexander von Humboldt Foundation and the program of the Russian Government to support research conducted by leading scientists (grant no. 11.G34.31.0045) is gratefully acknowledged.

\section{Notes and references}

1 K. H. Hausser and D. Stehlik, Adv. Magn. Reson., 1968, 3, 79. 2 T. Maly, G. T. Debelouchina, V. S. Bajaj, K. N. Hu, C. G. Joo, M. L. Mak-Jurkauskas, J. R. Sirigiri, P. C. A. van der Wel,
J. Herzfeld, R. J. Temkin and R. G. Griffin, J. Chem. Phys., 2008, 128, 052211.

3 C. Griesinger, M. Bennati, H.-M. Vieth, C. Luchinat, G. Parigi, P. Höfer, F. Engelke, S. J. Glaser, V. Denysenkov and T. F. Prisner, Prog. Nucl. Magn. Reson. Spectrosc., 2012, 64, 4-28.

4 K. M. Salikhov, Y. N. Molin, R. Z. Sagdeev and A. L. Buchachenko, Spin Polarization and Magnetic Effects in Chemical Reactions, Elsevier, Amsterdam, 1984.

5 J. Natterer and J. Bargon, Prog. Nucl. Magn. Reson. Spectrosc., 1997, 31, 293-315.

6 S. B. Duckett and R. E. Mewis, Acc. Chem. Res., 2012, 45, 1247-1257.

7 R. A. Green, R. W. Adams, S. B. Duckett, R. E. Mewis, D. C. Williamson and G. G. R. Green, Prog. Nucl. Magn. Reson. Spectrosc., 2012, 67, 1-48.

8 H.-M. Vieth, V. Macho and D. Stehlik, Chem. Phys. Lett., 1979, 60, 368-371.

9 D. Stehlik and H.-M. Vieth, in Pulsed Magnetic Resonance: NMR, ESR, and Optics: a Recognition of E. L. Hahn, ed. D. M. S. Bagguley, Oxford University Press, Oxford, 1992, p. 446.

10 W. Happer, Rev. Mod. Phys., 1972, 44, 169.

11 C. R. Bowers and D. P. Weitekamp, J. Am. Chem. Soc., 1987, 109, 5541-5542.

12 M. G. Pravica and D. P. Weitekamp, Chem. Phys. Lett., 1988, 145, 255-258.

13 K. L. Ivanov, H.-M. Vieth, K. Miesel, A. V. Yurkovskaya and R. Z. Sagdeev, Phys. Chem. Chem. Phys., 2003, 5, 3470-3480.

14 M. Goldman and H. Jóhannesson, C. R. Phys., 2005, 6, 575-581.

15 M. Goldman, H. Jóhannesson, O. Axelsson and M. Karlsson, C. R. Chim., 2006, 9, 357-363.

16 E. Y. Chekmenev, V. A. Norton, D. P. Weitekamp and P. Bhattacharya, J. Am. Chem. Soc., 2009, 131, 3164-3165.

17 C. Bretschneider, A. Karabanov, N. C. Nielsen and W. Köckenberger, J. Chem. Phys., 2012, 136, 094201.

18 C. Cai, A. M. Coffey, R. V. Shchepin, E. Y. Chekmenev and K. W. Waddell, J. Phys. Chem. B, 2013, 117, 1219-1224.

19 L. Buljubasich, I. Prina, M. B. Franzoni, K. Münnemann, H. W. Spiess and R. H. Acosta, J. Magn. Reson., 2013, 230, 155-159.

20 J. F. Dechent, L. Buljubasich, L. M. Schreiber, H. W. Spiess and K. Münnemann, Phys. Chem. Chem. Phys., 2012, 14, 2346-2352.

21 K. L. Ivanov, M. V. Petrova, N. N. Lukzen and R. Z. Sagdeev, Dokl. Phys. Chem., 2009, 427, 121-124.

22 T. Ratajczyk, T. Gutmann, S. Dillenberger, S. Abdulhussaein, J. Frydel, H. Breitzke, U. Bommerich, T. Trantzschel, J. Bernarding, P. C. M. M. Magusin and G. Buntkowsky, Solid State Nucl. Magn. Reson., 2012, 43-44, 14-21.

23 K. L. Ivanov, A. V. Yurkovskaya and H.-M. Vieth, J. Chem. Phys., 2008, 128, 154701.

24 E. A. Nasibulov, A. N. Pravdivtsev, A. V. Yurkovskaya, N. N. Lukzen, H.-M. Vieth and K. L. Ivanov, Z. Phys. Chem., 2013, 227, 929-953. 
25 R. R. Ernst, G. Bodenhausen and A. Wokaun, Principles of Nuclear Magnetic Resonance in One and Two Dimensions, Clarendon Press, Oxford, 1987.

26 S. Schäublin, A. Höhener and R. R. Ernst, J. Magn. Reson., 1974, 13, 196-216.

27 S. E. Korchak, K. L. Ivanov, A. V. Yurkovskaya and H. M. Vieth, Phys. Chem. Chem. Phys., 2009, 11, 11146-11156.

28 M. Carravetta and M. H. Levitt, J. Am. Chem. Soc., 2004, 126, 6228-6229.
29 G. Pileio, Prog. Nucl. Magn. Reson. Spectrosc., 2010, 56, 217-231. 30 M. H. Levitt, Annu. Rev. Phys. Chem., 2012, 63, 89-105.

31 P. R. Vasos, A. Comment, R. Sarkar, P. Ahuja, S. Jannin, J. P. Ansermet, J. A. Konter, P. Hautle, B. van den Brandt and G. Bodenhausen, Proc. Natl. Acad. Sci. U. S. A., 2009, 106, 18469-18473.

32 L. T. Kuhn, U. Bommerich and J. Bargon, J. Phys. Chem. A, 2006, 110, 3521-3526.

33 A. S. Kiryutin, K. L. Ivanov, A. V. Yurkovskaya, R. Kaptein and H.-M. Vieth, Z. Phys. Chem., 2012, 226, 1343-1362. 